\title{
Mégara Hyblaea, campagnes 2020
}

Henri Tréziny, Jean-Christophe Sourisseau, Reine-Marie Bérard, Frédéric Mège et Chloé Chaigneau

\section{(2) OpenEdition Journals}

Édition électronique

URL : https://journals.openedition.org/baefe/3173

DOI : $10.4000 /$ baefe.3173

ISSN : 2732-687X

Éditeur

ResEFE

\section{Référence électronique}

Henri Tréziny, Jean-Christophe Sourisseau, Reine-Marie Bérard, Frédéric Mège et Chloé Chaigneau, « Mégara Hyblaea, campagnes 2020 » [notice archéologique], Bulletin archéologique des Écoles françaises à l'étranger [En ligne], Italie, mis en ligne le 21 juillet 2021, consulté le 23 juillet 2021. URL: http://journals.openedition.org/baefe/3173 ; DOI : https://doi.org/10.4000/baefe.3173

Ce document a été généré automatiquement le 23 juillet 2021.

\section{cc) $(1) \&$}

Le Bulletin archéologique des Écoles françaises à l'étranger est mise à disposition selon les termes de la Licence Creative Commons Attribution - Pas d'Utilisation Commerciale - Pas de Modification 4.0 International. 


\title{
Mégara Hyblaea, campagnes 2020
}

\author{
Henri Tréziny, Jean-Christophe Sourisseau, Reine-Marie Bérard, Frédéric \\ Mège et Chloé Chaigneau
}

\section{NOTE DE L'AUTEUR}

Autorité nationale présente : Lorenzo Guzzardi, Parco archeologico di Leontinoi. Composition de l'équipe de terrain : Ont participé à ces campagnes Reine-Marie Bérard (CNRS, CCJ) ; Chloé Chaigneau (Université Paris 1 Panthéon-Sorbonne, ArScAn) ; Rosa Anna Corsaro (Institut National de Géophysique et de Volcanologie, Catane) ; Frédéric Mège (AMU, CCJ) ; Jean-Christophe Sourisseau (AMU, CCJ).

Partenariats institutionnels : EFR (École française de Rome), CJB (Centre Jean Bérard), AMU (Aix Marseille Université), CNRS (Centre National de la Recherche Scientifique), CCJ (Centre Camille Jullian), Unité Mixte de recherche 7299 AMU/CNRS, Soprintendenza di Siracusa, Parco archeologico di Leontinoi.

Établissements porteurs de l'opération : EFR, CNRS, Aix Marseille Université

\section{Chroniques de l'EFR :}

https://journals.openedition.org/cefr/4311

L'année 2020 a été, pour les études mégariennes comme pour le reste du monde, largement perturbée par la pandémie de CoVid 19. La quatrième campagne de fouilles, prévue pour une durée de cinq semaines en avril-mai 2020 dans la zone du sanctuaire du Nord-Ouest, a dû être annulée en raison des restrictions de circulation en Europe. Dans l'impossibilité de reprogrammer cette mission dans le courant d'une année très troublée, la reprise des fouilles dans ce secteur a été reportée au printemps 2021. Il a cependant été possible de réaliser cette année deux missions, la première de prospections géophysiques, la seconde d'étude du mobilier, et de poser les bases d'un nouveau programme de recherche pour les années à venir. 


\section{Les prospections géophysiques}

2 L'impossibilité d'organiser une campagne de fouilles en 2020 a paradoxalement créé une opportunité : celle de dégager les fonds nécessaires à la réalisation de prospections géophysiques, confiée à la société Géocarta. Elles avaient pour ambition de vérifier l'extension vers le Sud des rues E (identifiées sur le plateau Nord de Mégara Hyblaea dans les fouilles 2017-2019), et l'hypothèse, actuellement retenue, que ces rues s'inscrivaient dans un plan d'extension de l'habitat mégarien dans tout l'espace clôturé par la fortification.

Or, le réseau des rues nord-sud appelées $\mathrm{E}$, déjà bien connu dans la partie nord-ouest du site, se retrouve avec une grande netteté dans la partie sud-ouest, jusqu'à la fortification méridionale (fig. 1). Une rue plus large, orientée ouest-sud-ouest - estnord-est, coupe le réseau E. Elle semble en relation à l'ouest avec la porte Sud-Ouest et porter vers l'est en direction de la dépression de l'Arenella qui sépare les plateaux Nord et Sud de Mégara Hyblaea. La distance entre cette rue et la rue est-ouest B est de l'ordre de $200 \mathrm{~m}$, sans rue intermédiaire, ce qui surprend. Vers l'est, en direction de la voie ferrée et sur le plateau Sud, un réseau de rues d'une autre orientation semble apparaître ${ }^{1}$, mais cela demande confirmation. La campagne s'étant déroulée très tard, l'interprétation de ces images est encore en cours et nous nous limitons ici à une présentation rapide, en renvoyant pour plus de détail à la prochaine contribution du Bulletin, en 2021.

Fig. 1. Vue d'ensemble du site de Mégara Hyblaea avec les zones ayant fait l'objet de prospections géophysiques et gros plan sur les zones prospectées en 2021.

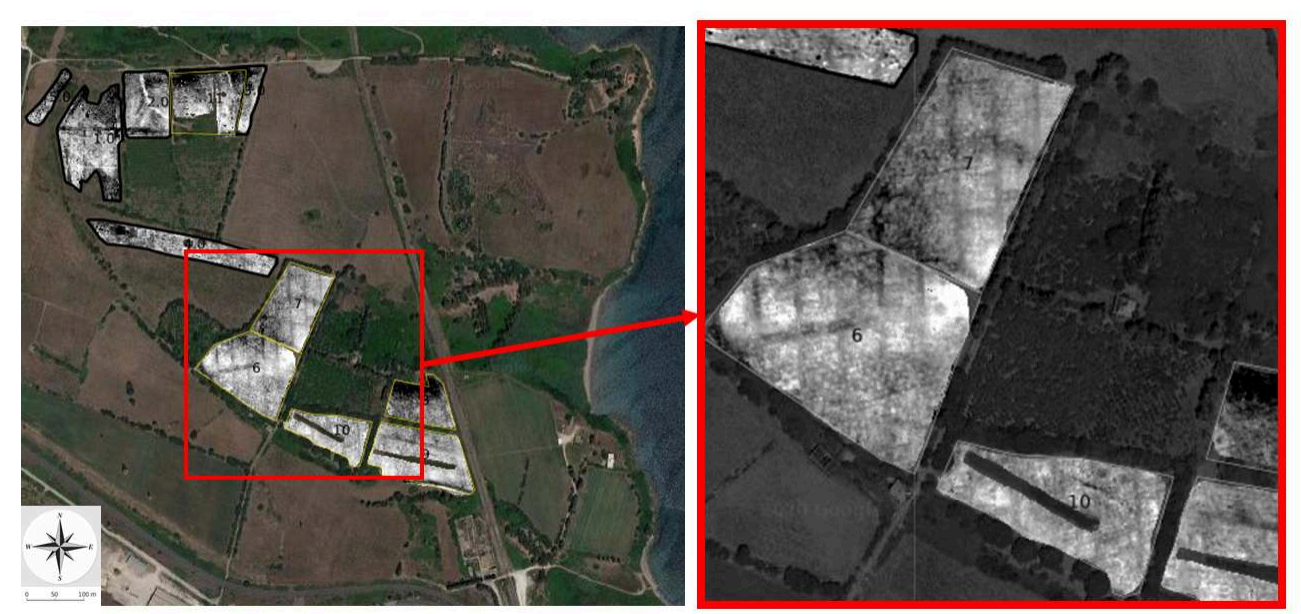

Google Map, Geocarta.

\section{La mission d'étude}

4 Malgré les conditions difficiles de circulation et de travail à l'automne 2020, il a été possible de poursuivre les études en cours à l'occasion d'une mission d'étude menée en septembre-octobre. 
5 En septembre, Frédéric Mège a pu mener la dernière mission d'étude de son programme de recherche $\mathrm{CaF}$ "Concretes as Floors », en cours depuis avril 2019 dans le cadre d'un contrat de chercheur à la Freie Universität Berlin et d'un financement de la Deutsche Forschungsgemeinschaft. Ce programme avait pour but d'étudier la composition physico-chimique de certains bétons de Mégara et de Sélinonte, majoritairement des bétons de tuileau, et d'en dater plusieurs à partir du $\mathrm{C}^{14}$ piégé dans la matrice au moment de la construction. Sept prélèvements ont été effectués en septembre 2020 par Raffaella D'Amico, restauratrice au musée de Lentini. Ils ont été complétés par des fragments provenant de trois sols conservés dans les réserves du site et utilisés dans une précédente étude ${ }^{2}$. Ces dix échantillons ont été examinés par Arnaud Coutelas (Arkemine SA, France), dont plusieurs en lames minces par microscope optique polarisée (fig. 2), l'un d'entre eux ayant également bénéficié d'analyses par MEB (Microscope Électronique à Balayage). Deux ont été datés par Alf Lindroos (Åbo Akademi University, Finlande) et Jesper Olsen (Aarhus Universitet, Danemark) au moyen d'une SMA (Spectrométrie de Masse par Accélérateur).

Fig. 2. Lames minces des bétons mégariens.

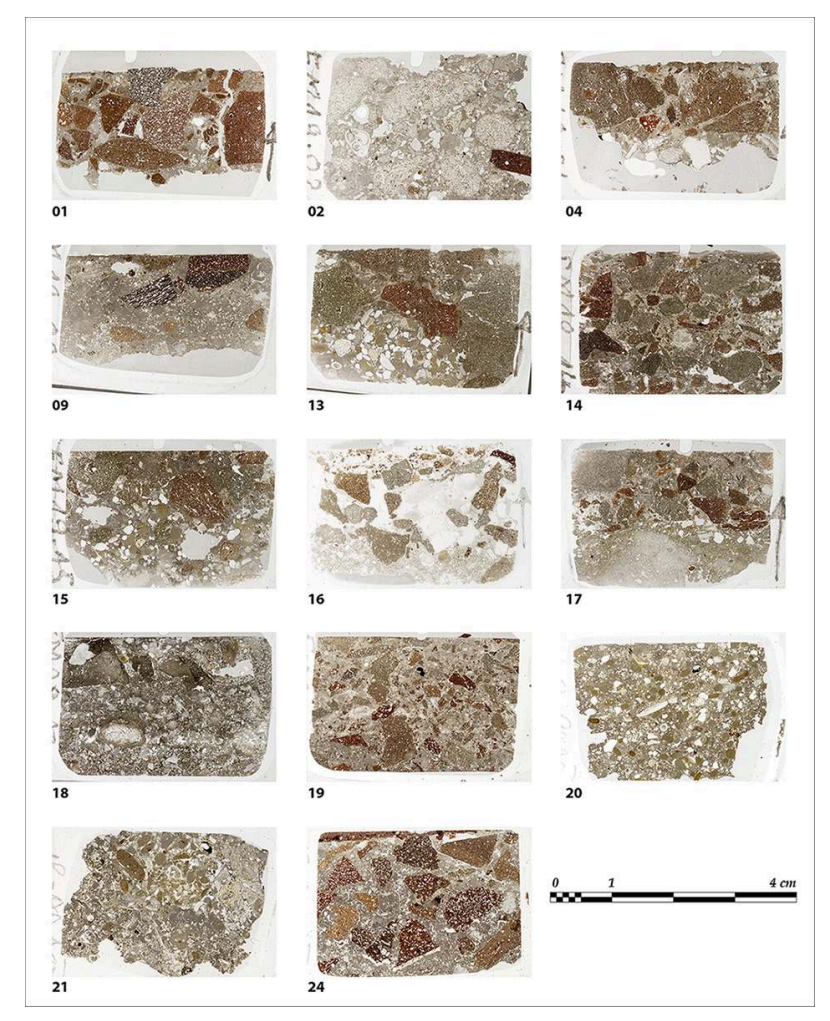

A. Coutelas, ArkeMine.

Les analyses pétrographiques ont montré que tous les bétons de Mégara ont été réalisés avec de la chaux aérienne pure, en proportion de $1 / 3$ ou $1 / 4$, avec des agrégats constitués soit de graviers et de galets de calcaire, soit de sable calcaire fin ou grossier associé à quelques grains siliceux, éventuellement complétés par du tuileau. Ce dernier provenait du broyage de terres cuites architecturales plutôt que de poteries diverses. Tous ces matériaux étaient d'origine locale, ce qui est la norme pour les bétons et mortiers anciens. 
7 La structure des bétons de Mégara et leurs compositions les rapprochent fortement de ce que l'on connaît dans le monde romain, l'un d'entre eux ayant d'ailleurs été daté du $\mathrm{II}^{\mathrm{e}} \mathrm{s}$. av. J.-C. ; l'analyse de ce béton de tuileau, qui appartenait aux bains hellénistiques, complète notre connaissance de cet édifice particulier ${ }^{3}$. Cela semble par ailleurs confirmer certaines hypothèses quant à l'apparition relativement tardive des bétons à Mégara ${ }^{4}$. L'ensemble de ces données sera présenté dans une publication prochaine où elles seront confrontées, et c'est là tout le sens du projet $\mathrm{CaF}$, aux résultats issus d'études plus traditionnelles, notamment stylistiques et architecturales.

8 En octobre 2020, Jean-Christophe Sourisseau et Reine-Marie Bérard ont par ailleurs achevé l'étude et le dessin du mobilier céramique significatif de la fouille 2019 et complété la documentation des missions antérieures. Il a ainsi été possible d'affiner la datation des secteurs en cours de fouille et la compréhension des résultats obtenus jusqu'à présent. L'achèvement et la mise au nette de la documentation relative à toutes les campagnes précédentes ont ainsi permis de préparer au mieux la reprise des fouilles en 2021, en redimensionnant le programme des opérations pour s'adapter aux contraintes inattendues rencontrées en 2020.

9 Ce séjour a également été l'occasion de prendre contact avec le nouveau directeur du musée archéologique Paolo Orsi de Syracuse, Carlo Staffile (qui a succédé à Calogero Rizzuto, disparu brusquement en février 2020), ainsi qu'avec la nouvelle directrice de l'unité opérative du musée, Rosa Lanteri. Cette dernière a notamment autorisé la réalisation d'une série de clichés du matériel de certaines tombes de la nécropole Ouest de Mégara Hyblaea par la photographe du musée, Germana Gallitto. Ces photographies ont permis à R.-M. Bérard de continuer ses recherches sur les nécropoles mégariennes en abordant un aspect encore peu connu de la typologie des tombes : les architectures funéraires en bois, documentées par la présence d'ensembles de clous dans de nombreuses tombes. 
Fig. 3. Clous de bronze, chevilles de fer et mobilier métallique de la tombe 361, Nécropole Ouest.

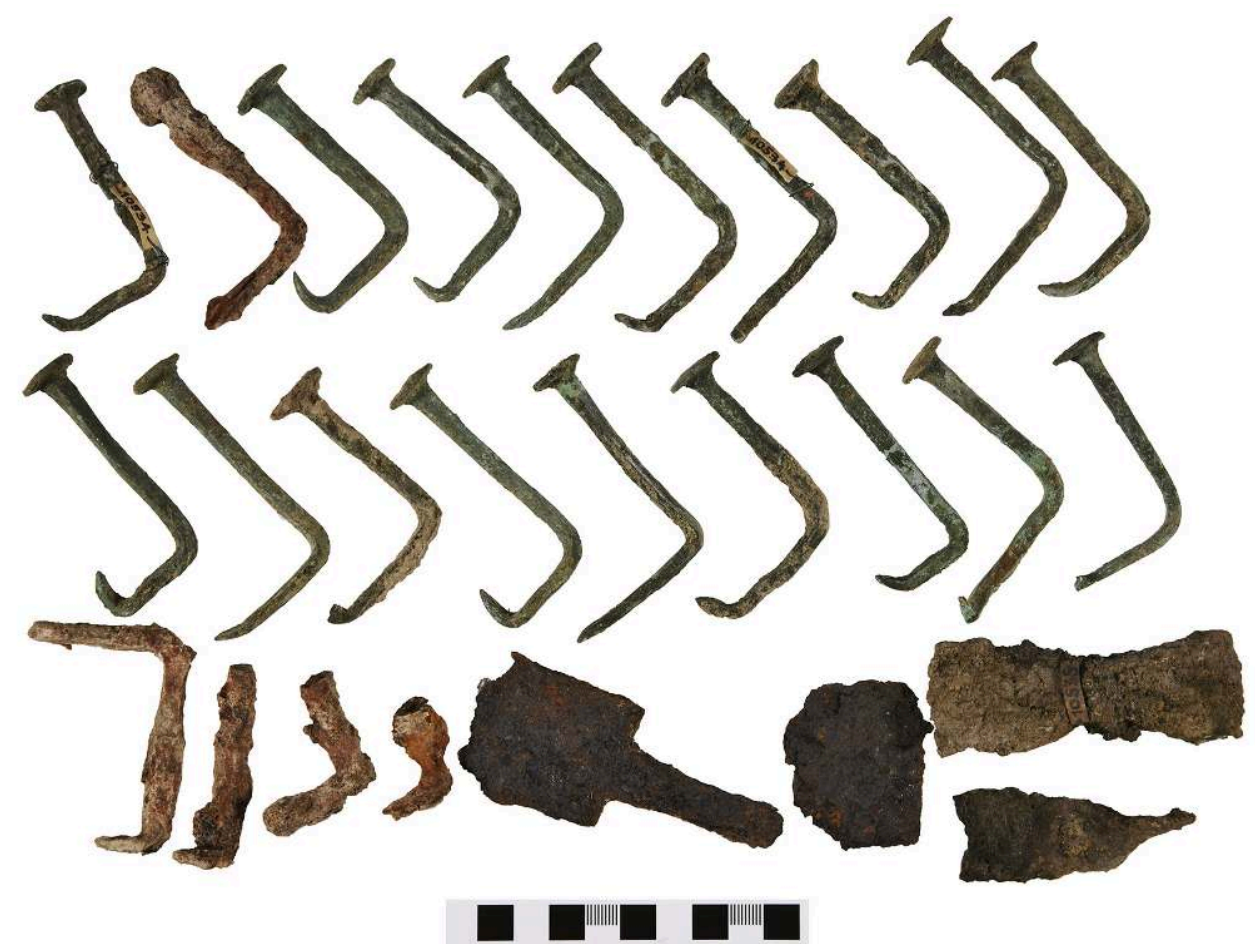

G. Gallitto, Museo Archeologico Regionale Paolo Orsi, Siracusa

10 Chloé Chaigneau a, quant à elle, procédé à l'inventaire et au classement de l'ensemble des meules grecques et romaines de Mégara Hyblaea qui font l'objet de son doctorat. Avec l'accord du directeur du Parc Archéologique de Leontinoi, Lorenzo Guzzardi, et avec le concours de la géologue Rosa Anna Corsaro (Institut National de Géophysique et de Volcanologie, Catane), elle a procédé à l'échantillonnage de 57 des 133 meules et fragments de meules inventoriés, afin de déterminer l'origine des roches employées pour leur fabrication. 
Fig. 4. Principaux types de roches meulières utilisées à Mégara Hyblaea.
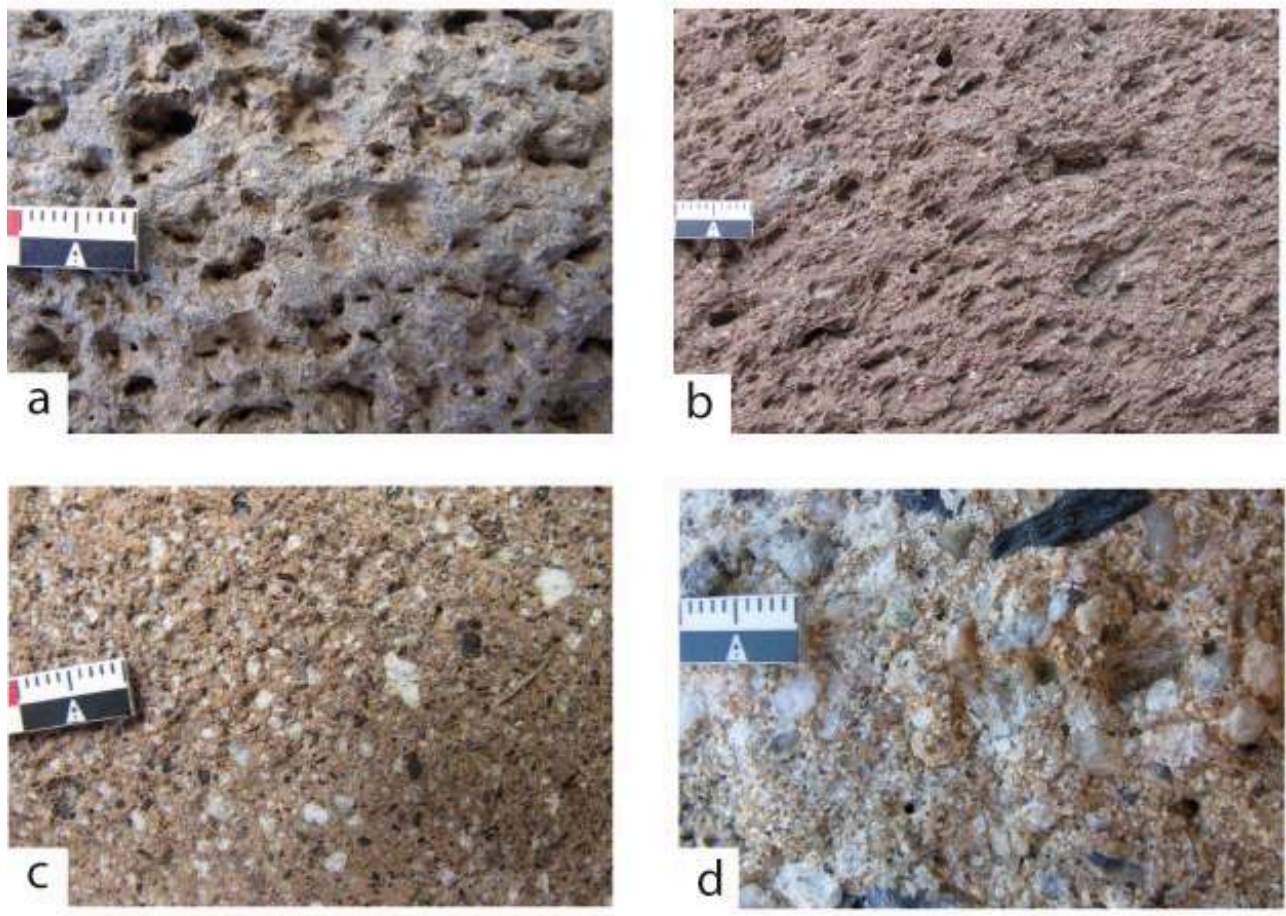

Ch. Chaigneau, Paris 1, ArScAn.

11 Une première observation macroscopique a d'ores et déjà permis de distinguer un type de roche sédimentaire, minoritaire dans les roches meulières mégariennes (fig. 3d) et trois principaux types de roches volcaniques: un basalte gris foncé à larges vacuoles, présent notamment en Sicile, mais non spécifique (fig. 3a) ; une roche plus rouge, très altérée, aux vacuoles particulièrement fines (fig. 3b), identifiée comme de la rhyolite de Sardaigne par O. Williams-Thorpe dans les années 1980, mais qui pourrait en réalité aussi être un basalte altéré sicilien ; une roche extra-sicilienne qui pourrait provenir du golfe Saronique en Grèce (fig. 3c). Les analyses devraient permettre de vérifier et préciser ces hypothèses. L'analyse pétrographique des lames minces sera effectuée à Urbino par A. Renzulli et P. Santi. Une sélection des échantillons les plus significatifs fera ensuite l'objet d'analyses géochimiques auprès du laboratoire ActLab (Canada).

Cette mission a enfin été l'occasion de travaux de valorisation et réorganisation du site et des réserves. On a ainsi commencé la mise à jour de l'inventaire du matériel archéologique conservé dans les magasins de Mégara Hyblaea, ainsi que des éléments architecturaux, meules et grands contenants conservés à l'extérieur des magasins. On a aussi pu constater l'aboutissement du projet de rénovation du parcours de visite du site, pour lequel Henri Tréziny avait rédigé de nouveaux panneaux, désormais accessibles en italien, en anglais et en braille (avec des plans en relief). La signalétique, reprenant le code couleur établi par Georges Vallet pour la délimitation des édifices sur le terrain, permet de distinguer les bâtiments d'époque archaïque (rouges) et hellénistique (verts). L'orientation systématique des panneaux en fonction de l'orientation des bâtiments facilite le repérage dans l'espace et la compréhension des vestiges. Cette nouvelle signalétique constitue ainsi une importante avancée pour la mise en valeur du site. 
Fig. 5. La nouvelle signalétique mise en place sur le site.

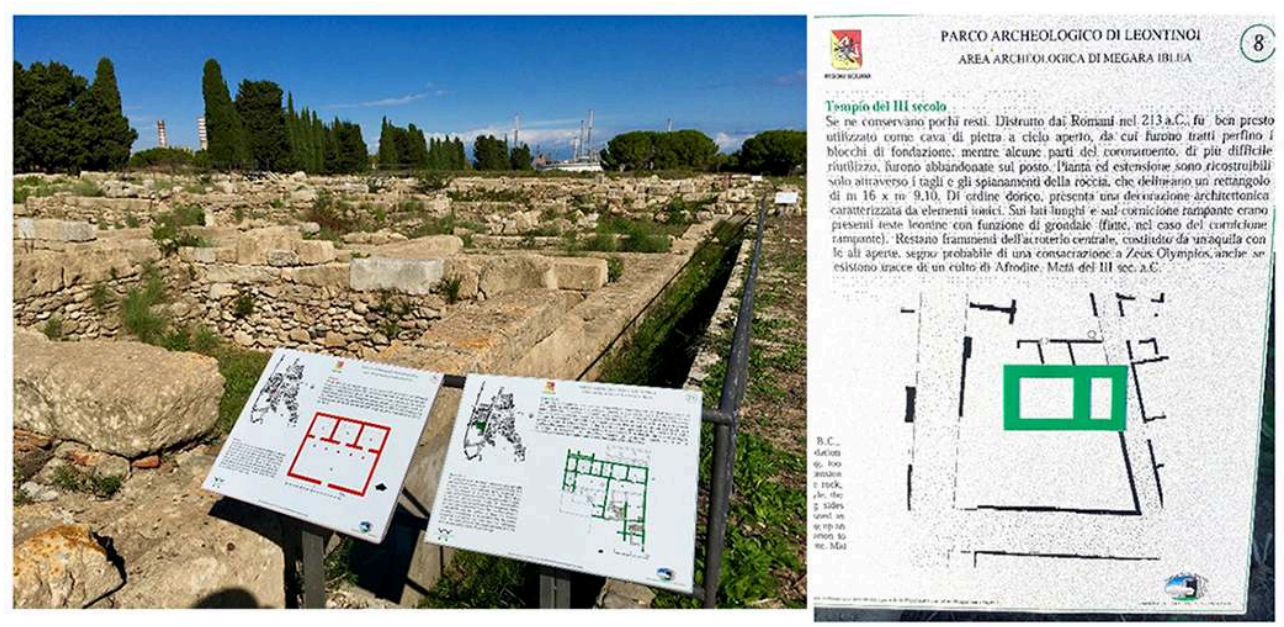

En rouge les bâtiments d'époque archaïque, en vert d'époque hellénistique.

R.-M. Bérard, CNRS, CCJ.

\section{Préparation d'un nouveau programme de recherche}

13 Enfin, cette mission a permis de poser les bases d'un nouveau programme de recherche envisagé dans la zone de l'Arenella - nom donné à la plage et, par extension, à la dépression qui se situe entre les deux plateaux Nord et Sud de Mégara Hyblaea. En 1952, 1962 et 1978, G. Vallet et F. Villard y avaient mené une série de petits sondages ${ }^{5}$, mettant au jour un mur en grand appareil, ainsi qu'une base inscrite archaïque $^{6}$ et une série de tombes hellénistiques. En 1989-1990 et 1992, deux campagnes de fouilles d'ampleur furent menées dans la zone par la Surintendance, sous la direction de Giuseppe Voza et G. Vallet ${ }^{7}$, mettant au jour de nouveaux ensembles architecturaux et un ensemble de tombes, parmi lesquelles figuraient à la fois des tombes hellénistiques et des tombes archaïques anciennes (vers 700). Cette zone, probablement suburbaine dans un premier temps avant d'être, peut-être, intégrée au tracé du rempart, est donc particulièrement intéressante pour comprendre les phases successives d'occupation et d'urbanisation de Mégara Hyblaea, mais elle reste, à ce jour, assez mal connue. C'est pourquoi nous envisageons un nouveau programme de recherche dans cette importante réserve archéologique, dont nous souhaiterions explorer la partie amont, entre la fouille de 1990-1992 et la voie ferrée Catane-Syracuse, qui traverse Mégara Hyblaea.

14 La mission d'étude d'octobre 2020 a ainsi été l'occasion de repérages topographiques dans la zone en compagnie de L. Guzzardi, responsable de l'aire archéologique de Mégara. Celui-ci s'est montré très favorable au projet et a commencé à envisager les opérations nécessaires au dégagement et à la mise en sécurité de la zone. La Surintendante de Syracuse, Donatella Aprile a également donné son accord de principe et autorisé l'accès aux archives textuelles et photographiques relatives aux opérations menées en 1990-1992 dans ce secteur. Il a en outre été possible d'avoir accès aux carnets de fouilles numérisés, dont on a commencé la transcription, ainsi qu'à l'ensemble des photographies relatives à ces fouilles. La mission d'étude 2020 a enfin 
permis à J.-C. Sourisseau et R.-M. Bérard d'entreprendre un premier inventaire sommaire des caisses de matériel provenant de ces fouilles (107 caisses pour 1990 ; 211 caisses pour 1992), conservées dans les magasins de Mégara. Les premiers éléments observés permettent d'établir une occupation de la zone sur la longue durée, de la fin $\mathrm{du} \mathrm{VIII}^{\mathrm{e}}$ siècle à l'époque hellénistique, révélant une aire complexe dont plusieurs éléments semblent indiquer qu'elle aurait pu abriter un sanctuaire.

Les caisses d'ossements provenant des tombes archaïques et hellénistiques de la zone ayant été conservées, R.-M. Bérard a pu réaliser une première étude anthropologique de ces restes osseux (fig. 6), qui permettra, à terme, de proposer une étude archéologique et anthropologique complète de cet ensemble de sépultures déjà brièvement présentées dans Mégara $7^{8}$.

Fig. 6. Ossements provenant des tombes de l'Arenella.

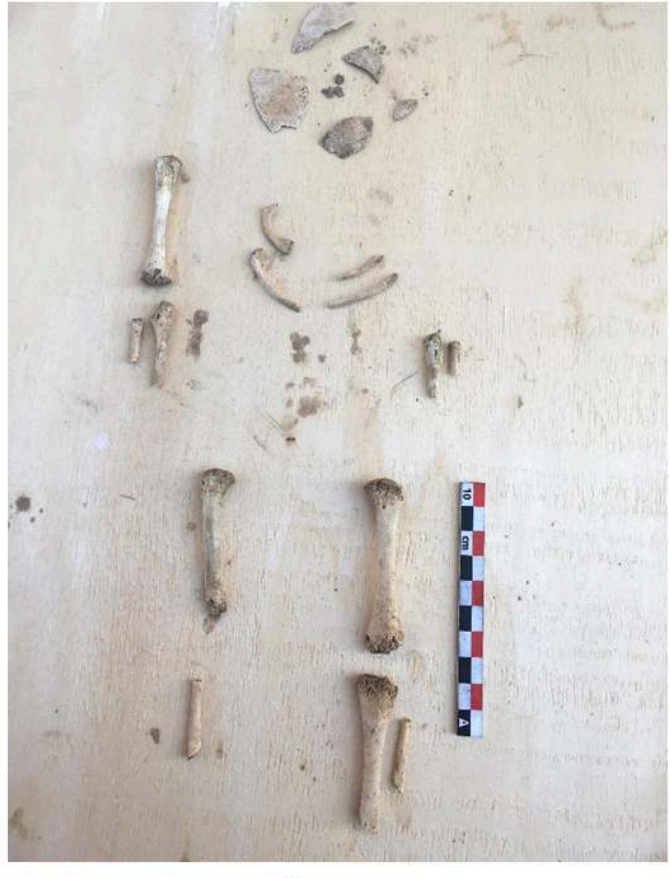

a

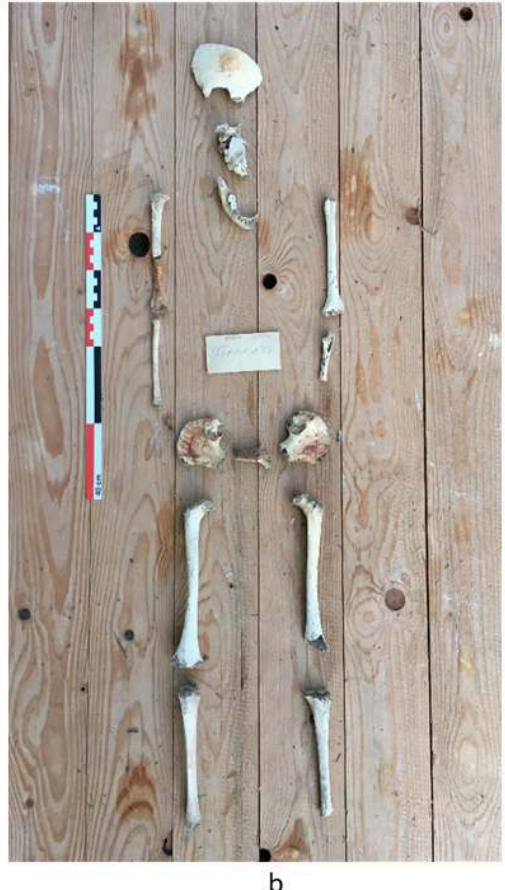

a. tombe 11, enfant mort en période périnatale ; b. tombe 13, enfant âgé de 6 à 8 ans au moment du décès.

R.-M. Bérard, CNRS, CCJ.

\section{BIBLIOGRAPHIE}

GRAS, TRÉZINY, BROISE 2004

Michel Gras, Henri Tréziny, Henri Broise, Mégara Hyblaea, 5. La ville archaïque : l'espace urbain d'une cité grecque de Sicile orientale, Rome, École française de Rome, 2004. 
MANNI PIRAINO 1979

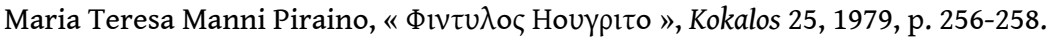

MÈGE 2019

Frédéric Mège, « Cement Floors of Megara Hyblaea. A Sicilian Perspective », in S. Bouffier,

I. Fumadó Ortega (éd.), Mortiers et hydraulique en Méditerranée antique, Aix-en-Provence, 2019,

p. 75-86.

MÈGE 2021

Frédéric Mège, Contribution à l'étude du fait urbain en Sicile hellénistique. L'habitat à Mégara Hyblaea aux IV et III siècles av. J.-C., Naples, Centre Jean Bérard, 2021.

TRÉZINY 2018

Henri Tréziny, Mégara Hyblaea 7. La ville classique, hellénistique et romaine, Rome, École française de Rome, 2018.

VALLET 1993

Georges Vallet, «Chronique des activités de l’École française de Rome en 1992 : Mégara

Hyblaea », Mélanges de l'École française de Rome. Antiquité 105-1, 1993, p. 462-470.

VILLARD, VALLET 1954

François Villard, Georges Vallet, « Mégara Hyblaea IV. La campagne de 1952 », Mélanges

d'archéologie et d'histoire 66, 1954, p. 13-38.

VOZA 1993

Giuseppe Voza, « Attività archeologica della Soprintendenza archeologica di Siracusa e Ragusa », Kokalos 39-40, 2, 1993, p. 1289-1293.

\section{Publications relatives au programme MEGA parues en 2020 (selon la nomenclature de l'HCERES)}

ACL

MÈGE et al. 2020

F. Mège, C. Belfiore, C. Monaco, A. Pezzino, H. Tréziny, Premier bilan des recherches préliminaires sur la pierre de construction à Mégara Hyblaea, MEFRA 2020-2, 2020.

TRÉZINY

H. Tréziny, Nouvelles données sur l'occupation de l'espace à Mégara Hyblaea à l'époque archaïque, Bulletin de la Société Française d'Archéologie Classique (XLIX, 2018-2019), dans Revue Archéologique 1, 2020, p. 143-149.

\section{C-ACTI}

BÉRARD 2020

R.-M. Bérard, Une autre façon de mourir ? Retour sur les pratiques funéraires de Mégara Nisaea et Mégara Hyblaea, dans M. Dana, M. Costanzi (dir.), Une autre façon d'être Grec : interactions et productions des Grecs en milieu colonial, Peeters Publishers (Colloquia Antica), Louvain, 2020, p. 333-350. 


\section{NOTES}

1. GRAS, TRÉZINY, BROISE 2004, fig. 170, rue nº 10 .

2. MÈGE 2019.

3. A propos des travaux récents sur cet édifice, voir notamment: https:// journals.openedition.org/cefr/1162.

4. MÈGE 2019 ; MÈGE 2021.

5. VILLARD, VALLET 1954, p. 31.

6. Celle de Phintylos fils d'Eugritos. Voir MANNI PIRAINO 1979, p. 256, pl. 14

7. VALLET 1993, p. 462-470 ; VOZA 1993, p. 1293-1294; GRAS, TRÉZINY, BROISE 2004, p. 288-292.

8. TRÉZINY 2018, p. 65-69.

\section{INDEX}

Année de l'opération : 2020

sujets https://ark.frantiq.fr/ark:/26678/pcrtse6crobzf3, https://ark.frantiq.fr/ark:/26678/ pcrtF9P4mbuyGk, https://ark.frantiq.fr/ark:/26678/pcrtWegewfitfX, https://ark.frantiq.fr/ark:/ 26678/pcrtQNxYz6P6Mh, https://ark.frantiq.fr/ark:/26678/pcrt1GqfoUzaZ3, https:// ark.frantiq.fr/ark:/26678/pcrtsIm3RuNMGu

Thèmes : EFR

\section{AUTEURS}

HENRI TRÉZINY

CNRS, AMU, CCJ

JEAN-CHRISTOPHE SOURISSEAU

AMU, CCJ

REINE-MARIE BÉRARD

CNRS, AMU, CCJ

FRÉDÉRIC MÈGE

AMU, CCJ

\section{CHLOÉ CHAIGNEAU}

ArScAn, Université Paris 1 